\title{
Catéter transhepático: última opción de acceso vascular en mujer de 26 años
}

\author{
Sonia Aznar Barbero, Ramón Bel Cegarra, Ma Magdalena Badallo Mira, David Pagán Escribano, Asensio \\ Bartolomé García Nicolás, Josefina Chica Arellano*
}

Enfermeros de la unidad de diálisis del Hospital Universitario Santa Lucía, Cartagena. * Supervisora de la Unidad de Hemodiálisis

\section{Introducción}

El acceso vascular (AV) es la base fundamental de todo tratamiento extracorpóreo de diálisis ${ }^{1}$, su funcionamiento adecuado influye directamente en la calidad de vida del paciente y en su morbi-mortalidad.

EI AV vascular ideal debe permitir el abordaje seguro y continuado del sistema vascular, proporcionar flujos suficientes para alcanzar una dosis de diálisis adecuada y carecer de complicaciones ${ }^{2,3}$.

Según las guías SEN la primera opción de AV es la fístula arteriovenosa (FAV): nativa preferentemente, protésica, etc. Sin embargo, cuando estas posibilidades se agotan, se recurre a catéteres permanentes tunelizados ${ }^{4}$.

Actualmente el número de pacientes portadores de catéter venoso central se ha visto incrementado en las unidades de hemodiálisis superando con creces el porcentaje recomendado por las guías S.E. $N^{5}$ y KDOQI que lo sitúan por debajo del $10 \%$, esto puede ser debido al aumento de pacientes añosos y diabéticos y al fracaso recurrente de los accesos vasculares por disfunción, trombosis, infección, etc., que provoca un agotamiento irreversible de la red vascular.

El buen funcionamiento y perdurabilidad del AV depende de múltiples factores, pero ¿Qué ocurre cuando se ven agotados los accesos vasculares habituales en un paciente? ${ }^{7,8}$. La respuesta a esta pregunta sería recurrir a otros abordajes menos comunes con el fin de lograr un acceso vascular válido para hemodiálisis.

\section{Correspondencia: \\ Sonia Aznar Barbero \\ Agua del Molino n 35 \\ 30310. EL Bohío. Cartagena. Murcia \\ E-mail: soniazbar@hotmail.com}

Presentamos un caso clínico en el que destaca el intento desesperado de todo el equipo multidisciplinar por conseguir un acceso vascular de calidad que asegure la supervivencia de una paciente con problemas de socialización y de cumplimiento del tratamiento, hecho que influye negativamente en la perdurabilidad de dicho acceso.

\section{Objetivos}

1. Exponer nuestra experiencia con el catéter tunelizado transhepático.

2. Valorar si las venas hepáticas son una buena opción para proporcionar una hemodiálisis de calidad.

3. Valorar si se ven aumentadas las cargas de enfermería al tratar con un acceso vascular de uso poco habitual.

4. Conseguir una mayor colaboración de la paciente en el cuidado de su acceso vascular a través de la educación sanitaria.

5. Minimizar las complicaciones relacionadas con el AV derivadas del aumento de interés de la paciente por el cuidado del catéter.

6. Concienciar a todo el personal de enfermería del importante papel que juega en la supervivencia de los accesos vasculares de los pacientes.

\section{Caso clínico}

Mujer de 26 años con ERC secundaria a glomerulonefritis proliferativa extracapilar tipo I. Fumadora activa, Hipertensa aunque no precisa tratamiento farmacológico actualmente. Presenta enfermedad de Darier cutánea.

Recibió 14 sesiones de plasmaféresis tras su diagnóstico en 2006. Posteriormente abandonó las revisiones en consulta y finalmente acudió por urgencias 3 años después en situación de uremia terminal iniciando diálisis. 
La paciente da consentimiento expreso para la exposición de su caso.

\section{Historia del acceso vascular}

- Varios intentos de Fístula Arteriovenosa (FAV), todos ellos infructuosos: FAV radio-cefálica izquierda en 01-2009, húmero-cefálica izquierda en 03-09, radio-cefálica derecha en 04-09 y húmero-cefálica derecha en 05-11. Se le realizó un mapa vascular en el que no se evidenció ninguna vena apta en MMSS.

- Catéter venoso permanente yugular derecho, implantado el 14/01/09 y retirado el 05/11/09 por Endocarditis Tricuspídea secundaria a MARS.

- Varios catéteres temporales en ambas venas femorales retirados por trombosis o disfunción.

- Catéter permanente yugular izquierdo colocado el $16 / 11 / 09$ y retirado el $21 / 06 / 10$ por trombosis de la vena yugular izquierda.

- Catéter permanente femoral derecho colocado el 21/06/10 y retirado 4 días después por disfunción persistente.

- Tras ello se le realizó un estudio endovascular que mostraba oclusión completa de vena iliaca izquierda y de cava inferior, lo que inhabilitaba los accesos femorales. En lo que respecta a los troncos superiores la situación no es mejor pues existe oclusión de venas subclavias, yugular derecha, tronco braquio-cefálico izquierdo y cava superior. Ante esta situación se colocó catéter permanente por abordaje transhepático a través de vena suprahepática derecha, con extremo en aurícula derecha9. Se recambió el 03/07/2012 por disfunción. Desde entonces ha sufrido continuas infecciones que han obligado a instaurar tratamiento antibiótico casi de manera continua.

- En 12/2012 sufre extrusión espontánea colocándose catéter femoral provisional izquierdo.

- Se le realizó nuevo estudio de troncos venosos encontrando oclusión de vena iliaca con desviación del flujo por rama hipogástrica hasta iliaca común, de modo que no es subsidiaria de catéter de diálisis permanente por este acceso. La vena femoral común derecha se encontró permeable pero no se detectó flujo doppler en vena iliaca, probablemente por trombosis. Se realizaron flebografías de ambos brazos encontrando que el flujo va por colaterales a intercostales hasta acigos-hemiacigos de forma bilateral debido a la oclusión de las venas subclavias. Se descartó colocar un catéter por subclavia. Se realizó ecografía doppler de ambas yugulares visualizando la vena yugular izquierda permeable aunque es muy probable estenosis central del tronco braquiocefálico venoso izquierdo dada la flebografía de ese miembro y la vena yugular derecha ocluida crónica (no visible en la ecografía).

- El 17/01/2013 se le implantó nuevo catéter transhepático que fue recambiado el 20/03/2013 por disfunción. Este es su acceso actual y probablemente el único posible.

A todos estos problemas vasculares que ponen en riesgo su supervivencia se suma la situación social complicadísima que vive la paciente, ya que no recibe ningún apoyo familiar y se encuentra totalmente desmotivada presentando en la mayoría de ocasiones un comportamiento rebelde y de rechazo a cualquier cambio en sus opciones de tratamiento, hecho que dificulta enormemente la labor de enfermería en el ámbito de educación para la salud.

Cabe reseñar que la paciente rechaza iniciar cualquier tipo de tratamiento anticoagulante (se le ha propuesto tomar sintrom ${ }^{\circledR}$ en muchas ocasiones).

Por otra parte se descartó su inclusión en la lista de trasplante al no acudir a las reiteradas citas,

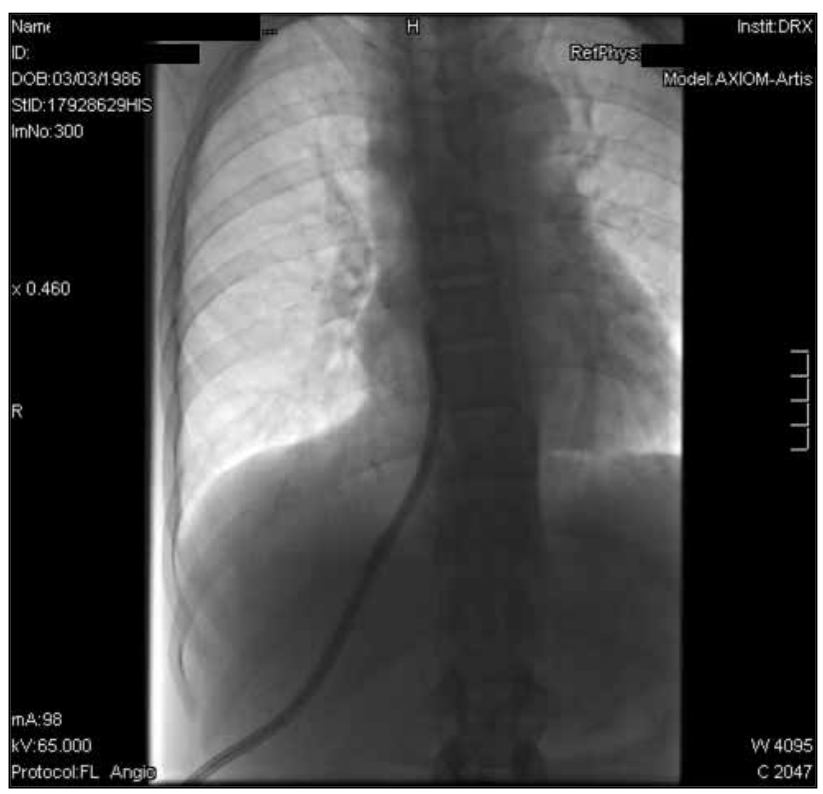

Figura 1. Control por imagen. 


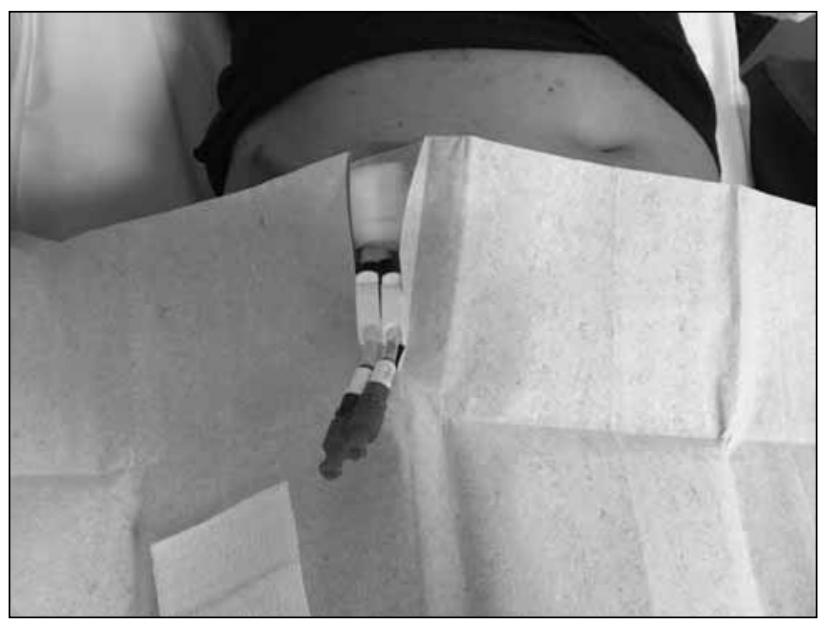

Figura 2. Preparación para la conexión.

autoexcluyéndose además como candidata a diálisis peritoneal dada su negativa a cumplir con las pautas de tratamiento propuestas por el personal médico y de enfermería.

En las figuras 1 y 2 se muestra el acceso actual de la paciente: el catéter transhepático.

\section{Cuidados de enfermería}

En cada sesión se informa a la paciente de los cuidados que debe tener para cuidar al máximo su AV haciendo especial hincapié en la importancia de mantener el apósito seco y limpio y de informar al personal de enfermería acerca de cualquier incidencia que note, especialmente si aparece dolor o calor en la zona de inserción y fiebre.

Todo el equipo de enfermería cumple con los protocolos de cura del orificio de salida del catéter, conexión y desconexión según los criterios aceptados en nuestra unidad de diálisis:

- La cura del orificio de salida del catéter (OSC) se realiza de forma aséptica con cloruro sódico al $20 \%$, clorhexidina alcohólica al $2 \%$ y gasas estériles, observando de forma exhaustiva la zona para detectar precozmente la aparición de eritema, secreción, etc. Después se cubre el orificio con un apósito estéril.

- El sellado de las ramas del catéter se realiza con Taurolock® y tapones tipo luer-lock. Desde el último recambio del catéter transhepático en la primera sesión de diálisis semanal se sella con Urokinasa $50.000 \mathrm{UI} / \mathrm{rama}$ como terapia fibrinolítica intraluminal según protocolo de nuestra unidad.

Se valora el funcionamiento adecuado del catéter en cada sesión de hemodiálisis alcanzando flujos sanguíneos adecuados superiores a $300 \mathrm{ml} / \mathrm{min}$ y Kt mayor de $45 \mathrm{~L}$.

En la figura 3 se muestran los datos obtenidos de las sesiones de diálisis desde enero de 2013.

\begin{tabular}{|c|c|c|c|c|c|c|c|}
\hline MESES & $\begin{array}{l}\text { Kt } \min / K t \\
\max (L)\end{array}$ & $\begin{array}{l}\text { Volumen } \\
\text { reinfusión } \\
\text { min/max } \\
\text { (L) }\end{array}$ & $\begin{array}{c}\text { Qb } \min / \\
\max (\operatorname{ml} / \\
\min )\end{array}$ & OSC & Tratamiento antibiótico & $\begin{array}{c}N^{0} \text { sesiones } \\
\text { con Urokinasa } \\
\text { (desobstrucción o } \\
\text { sellado) }\end{array}$ & $\begin{array}{c}\mathrm{N}^{0} \text { sesiones } \\
\mathbf{Q b}<300 \mathrm{ml} / \\
\mathrm{min}\end{array}$ \\
\hline ENERO & $47,6 / 59$ & $23 / 29,4$ & $300 / 400$ & Bien & $\begin{array}{l}\text { Levofloxacino } 500 \mathrm{mg} 1 \\
\text { dosis y } 250 \mathrm{mg} 6 \text { sesiones } \\
\text { mas }\end{array}$ & 0 & 0 \\
\hline FEBRERO & $45 / 57,7$ & $23 / 30 *$ & $230 / 400$ & Bien & - & 0 & 2 \\
\hline MARZO & $36 / 57,7$ & $23,46 / 30,7^{*}$ & $150 / 400$ & Bien & $\begin{array}{l}\text { Curoxima } 750 \mathrm{mg} 1 \text { dosis por } \\
\text { recambio catéter }\end{array}$ & 10 & 5 \\
\hline ABRIL & $47,9 / 58,8$ & $22,99 / 32,9$ & $300 / 400$ & Bien & - & 5 & 0 \\
\hline MAYO & $41 / 59,1$ & $23,25 / 29,98$ & $310 / 400$ & Bien & - & 4 & 0 \\
\hline JUNIO & $42,6 / 57,6$ & $16,9 / 27,45$ & $200 / 370$ & $\begin{array}{l}\text { Exudado } \\
\text { purulento }\end{array}$ & $\begin{array}{l}\text { Levofloxacino } 500 \mathrm{mg} 1 \\
\text { sesión y } 250 \mathrm{mg} 6 \text { sesiones } \\
\text { mas }\end{array}$ & 4 & 2 \\
\hline JULIO & $42,2 / 53,8$ & $21,06 / 30,2$ & $250 / 380$ & $\begin{array}{l}\text { Prurito y } \\
\text { eritema }\end{array}$ & $\begin{array}{l}\text { Vancomicina 500mg } 3 \\
\text { sesiones }\end{array}$ & 5 & 1 \\
\hline
\end{tabular}

Figura 3. Resumen de funcionamiento e incidencias del AV. 


\section{Discusión}

A lo largo de los últimos años hemos sido testigos del aumento en el número de pacientes que se dializan a través de catéter venoso central, dato que no es positivo ya que la presencia de un catéter venoso para HD se ha asociado con numerosas complicaciones que se traducen en una elevada morbimortalidad ${ }^{10}$.

El fracaso reiterado del AV se erige como factor de riesgo de mortalidad, debido al riesgo de coagulación, infección, subdiálisis y estenosis de vasos centrales, además de comportar un aumento del coste económicoll.

Este caso se encuentra en una situación crítica porque ha ido agotando paulatinamente los accesos vasculares y actualmente se dializa a través de un catéter transhepático, que es presumiblemente su última opción.

Probablemente la única alternativa para garantizar la supervivencia a no muy corto plazo sea seguir insistiendo en la promoción de hábitos saludables, la educación para la salud y mantener la colaboración con otros profesionales (psicóloga y trabajadora social de la asociación de enfermos renales) para ir logrando progresivamente un cambio en sus habilidades psicosociales de modo que presente una actitud más abierta y colaboradora que permitieran ampliar las opciones de tratamiento aún a día de hoy posibles.

\section{Conclusiones}

El catéter transhepático es una opción válida para proporcionar al paciente una diálisis adecuada.

> El personal de enfermería no encuentra diferencias en la manipulación y cuidado de éste tipo de catéter puesto que se aplican los mismos protocolos que para el resto de catéteres venosos centrales.

> La educación sanitaria se ha mostrado como herramienta insuficiente para modificar los hábitos higiénicos y conductuales de la paciente en relación a los cuidados de su acceso vascular.

$>$ El equipo de enfermería cumple con los protocolos de conexión, desconexión y cura del OSC y es consciente de la importancia de mantener una técnica aséptica para asegurar el buen funcionamiento del AV durante el máximo tiempo posible.

\section{Bibliografía}

1. J.R. Polo, J.M. López. Accesos vasculares para hemodiálisis. En: L. Hernando Avendaño. Nefrología Clínica Ed. Panamericana 2008, (19) p. 889-904.

2. Jimeno Martín I. et al. Indicaciones y tipos de accesos para Hemodiálisis. Dial Traspl. 2008;29(4): 173-6.

3. John T Daurgidas. Catéter venoso central para hemodiálisis. En: Manual de diálisis cap 6, p. 86-103.

4. J.A: Rodríguez Hernández et al. Guías SEN. Guías de acceso vascular en hemodiálisis. Nefrología 2005; V 25 (supl. 1): 1-174.

5. Guías SEN de Centros de Hemodiálisis. Nefrología 2006;6(Supl. 8).

6. National Kidney Foundation. KDOQI Clinical Practice Guidelines for Hemodialysis Adequacy, 2000. Am J Kidney Dis 2001;37:S7-S64.

7. L. Navarro, A. Reula, E.M. Martínez, A. Martínez, R. Ortells, A. Martí, C. Moreno. Dificultad para conseguir un acceso vascular para hemodiálisis: caso clínico: 9 años en Hemodiálisis, 15 accesos vasculares. Enferm Nefrol 2012: Julio-Septiembre; 15 (3): 219/221.

8. I. Crehuet, P. Méndez. Supervivencia de un catéter: un reto y un logro de enfermería. Rev Soc Esp Enferm Nefrol 2011; 14 (3): 189/194.

9. J. M. da Motta-Leal-Filho, F. C. Carnevale, F. Nasser, W. de Oliveira Sousa Junior, C. E. Zurstrassen, A. Mota, B. Boueri, G. Guido. Percutaneous transhepatic venous access for hemodialysis: an alternative route for patients with end-stage renal failure. $\mathrm{J}$ Vasc Bras. 2010; 9 (3): 131/136.

10. R. Roca Tey et al. El acceso vascular para hemodiálisis: la asignatura pendiente. Nefrología 2010;30(3):280-7.

11. E.Gruss, J. Portolés y cols. Repercusiones clínicas y económicas del uso de catéteres tunelizados de hemodiálisis en un área sanitaria. Nefrología 2009;29(2):123-129.

* En las sesiones en las que no se alcanzó un flujo sanguíneo superior a $250 \mathrm{ml} / \mathrm{min}$ se desactivó la hemodiafiltración on-line por hemoconcentración. 